\title{
Establishment and characterization of NCC-DMM1-C1, a novel patient-derived cell line of desmoplastic malignant pleural mesothelioma
}

\author{
REI NOGUCHI ${ }^{1}$, YUKI YOSHIMATSU ${ }^{1}$, TAKUYA ONO ${ }^{1}$, AKANE SEI ${ }^{1}$, NORIKO MOTOI ${ }^{2}$, \\ YASUSHI YATABE $^{2}$, YUKIHIRO YOSHIDA ${ }^{3}$, SHUNICHI WATANABE ${ }^{3}$ and TADASHI KONDO ${ }^{1}$ \\ ${ }^{1}$ Division of Rare Cancer Research, National Cancer Center Research Institute; \\ ${ }^{2}$ Department of Pathology and Clinical Laboratories, National Cancer Center Hospital; \\ ${ }^{3}$ Department of Thoracic Surgery, National Cancer Center Hospital, Chuo-ku, Tokyo 104-0045, Japan
}

Received June 6, 2021; Accepted October 27, 2021

DOI: $10.3892 / \mathrm{ol} .2021 .13182$

\begin{abstract}
Desmoplastic malignant pleural mesothelioma (DMM) is a rare histological variant of malignant pleural mesothelioma, which is a highly aggressive neoplasm of the mesothelium. DMM is associated with distant metastases and short survival.Effective treatments for DMM are not established and the development of histotype-tailored treatments is difficult due to the rarity of the disease. Although patient-derived cancer models are crucial tools for the development of novel therapeutics, they are difficult to obtain for DMM; no DMM cell lines or xenografts are available from public biobanks and only two cell lines have been reported. Thus, the present study aimed to establish a novel cell line of DMM as a resource for drug screening. A cell line of DMM was established, designated as NCC-DMM1-C1, using surgically resected tumor tissues from a 73-year-old male patient with DMM. Characteristics of NCC-DMM1-C1 cells were examined, such as growth, spheroid formation and invasion capability. Drug targets and anti-cancer drugs with anti-proliferative efficacy were examined using a comprehensive kinase activity assay and drug screening of 213 anti-cancer agents, respectively. NCC-DMM1-C1 exhibited fast growth, spheroid formation and invasion capability, suggesting that the NCC-DMM1-C1 cells retained the aggressive features of DMM. NCC-DMM1-C1 cells and the tumor tissue shared common activity profiles of kinases, which included FES, Wee1, platelet-derived growth factor receptor- $\beta$ and Src. The drug screening revealed that bortezomib, fostamatinib, gemcitabine, homoharringtonine
\end{abstract}

Correspondence to: Dr Tadashi Kondo, Division of Rare Cancer Research, National Cancer Center Research Institute, 5-1-1 Tsukiji, Chuo-ku, Tokyo 104-0045, Japan

E-mail: takondo@ncc.go.jp

Key words: desmoplastic malignant pleural mesothelioma, kinase, patient-derived cancer cells, pre-clinical study, high-throughput drug screening, comprehensive kinase activity assay and vinorelbine had anti-proliferative effects, which have not been previously reported for DMM. It was concluded that NCC-DMM1-C1 cells may be a useful tool for the study of DMM.

\section{Introduction}

Desmoplastic malignant pleural mesothelioma (DMM) is a rare histological variant of malignant pleural mesothelioma (MPM), accounting for 5-10\% of all cases of MPM. DMM is categorized as a subtype of sarcomatoid tumor (1) and characterized by dense, collagenized tissue ( $>50 \%)$ separated by atypical cells arranged in a storiform or 'patternless pattern' in the tumor specimen (2). The pathological diagnosis of DMM is difficult, with fibrous pleuritis and reactive mesothelial hyperplasia as potential differential diagnoses (3). The role of immunohistochemistry is important for the diagnosis of DMM and broad-spectrum staining of cytokeratins is crucial to diagnose DMM correctly. In addition, p16 deletion is useful to distinguish DMM from benign pleuritic (2). The characteristic computed tomography (CT) findings include unilateral pleural effusion, thickening of the mediastinal pleura, circumferential and nodular pleural thickening of $>1 \mathrm{~cm}$ and interlobar fissure thickening (4-6). MPM is refractory to chemotherapy and radiation therapy, and the combination of pemetrexed and cisplatin has become the standard first-line chemotherapy regimen for MPM based on the results of a randomized phase III trial (7). However, this regimen has only improved median survival from 9.3 months for treatment with cisplatin alone to 12.1 months (7) and it lacks the impact on survival that second-line therapy is expected to have (8). Thus, further investigations for novel therapeutics have long been desired for MPM and DMM (9).

Patient-derived cancer cells that retain the genetic and phenotypic profiles of their original tumor tissue are crucial for the elucidation of molecular mechanisms underlying the malignant features of tumors and for the development of novel therapies (10). In particular, patient-derived cell lines provide a model for screening numerous anti-cancer agents and investigate their modes of action in a high-throughput 
manner. Furthermore, cell lines have a utility in the development of predictive biomarkers and identification of therapeutic targets and previous studies have included numerous cell lines of various cancer types (11-13). Of note, the availability of cell lines is crucial for drawing the benefits of modern oncology technology. However, according to the largest cell line database, Cellosaurus (https://www.ncbi.nlm.nih. gov/pmc/articles/PMC5945021/), only two patient-derived cell lines of DMM, namely TCPH-MM02 (14) and TCPH-MM11 (15), have been reported in the literature and they are not available from any cell banks. The database of patient-derived xenografts (PDXs), PDXFinder (16), reveals no PDXs of DMM available from public biobanks. The paucity of adequate patient-derived cancer models is one factor hindering the development of novel therapies for DMM and the establishment of patient-derived DMM models is an urgent requirement.

In the present study, a novel cell line, designated NCC-DMM1-C1, was established from tumor tissue of DMM and characterized. Its utility in high-throughput screening of anti-cancer agents for anti-proliferative effects was then evaluated. To the best of our knowledge, the present study is the third report describing the establishment of a patient-derived cell line of DMM.

\section{Materials and methods}

Patient history. The patient was a 73-year-old male who visited the National Cancer Center Hospital (Tokyo, Japan) with a major symptom of progressive dyspnea. The patient had been exposed to asbestos from the age of 25 to 60 years. Enhanced CT detected pleural thickening and pleural effusion on the right side (Fig. 1A). Pathological diagnosis using pleural biopsy suggested DMM. Pleurectomy/decortication was performed and the definitive diagnosis from surgically resected tumor tissues was DMM (pT2 pN1 cM0 pStage II). The tumor tissue obtained at the time of surgery was used to establish the cell line. After seven months, multiple liver metastases occurred and the patient developed jaundice and died from pneumonia and respiratory failure due to DMM recurrence.

Cell culture procedure. Cell culture was performed according to a previous study by our group (17). Informed consent was obtained from the patient. In brief, the resected tumor tissue was mechanically dissected with scissors in tissue culture plates (Thermo Fisher Scientific, Inc.). The cells were maintained in Dulbecco's modified Eagle's medium (DMEM)/F12 (Sigma-Aldrich; Merck KGaA) supplemented with $10 \%$ heat-inactivated fetal bovine serum (FBS; Gibco; Thermo Fisher Scientific, Inc.), $100 \mathrm{U}$ penicillin $\mathrm{G}$ and $100 \mu \mathrm{g} / \mathrm{ml}$ streptomycin (Gibco; Thermo Fisher Scientific, Inc.). After reaching sub-confluence, the cells were treated with $0.25 \%$ trypsin-EDTA (Gibco; Thermo Fisher Scientific, Inc.) and transferred to a new tissue culture plate.

Authentication and quality control of the established cell line. The cell line was authenticated by examining the short tandem repeats (STRs) in 10 loci using the GenePrint 10 System (Promega Corporation), as previously described (17). Genomic DNA was extracted from the cells and the tumor tissue using

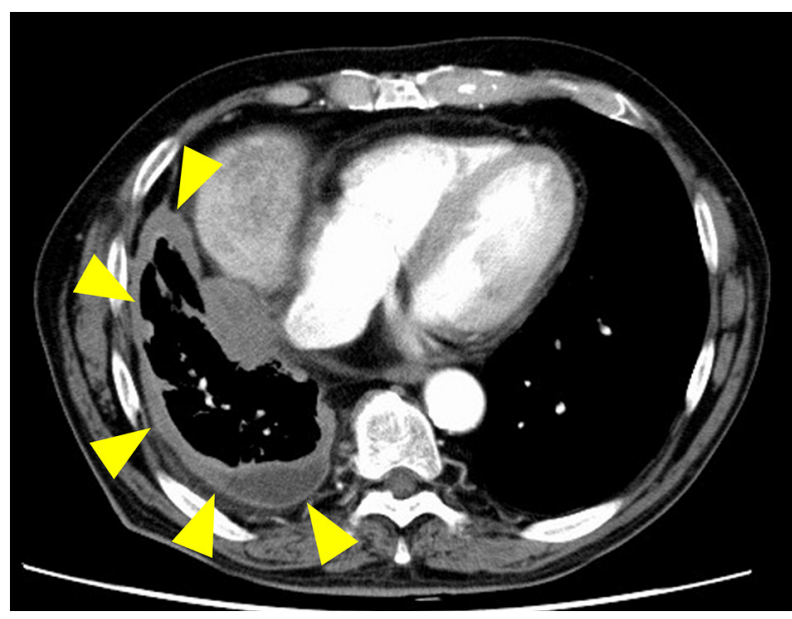

Figure 1. Clinical imaging. Computed tomography indicated irregular pleural thickening involving both parietal and visceral pleurae and pleural effusion in the right chest area. The yellow arrow indicates the right-sided desmoplastic malignant pleural mesothelioma.

a DNeasy Blood \& Tissue kit (Qiagen $\mathrm{GmbH}$ ) and quantified using a NanoDrop 8000 Spectrophotometer (Thermo Fisher Scientific, Inc.). STRs were amplified and sequenced using a 3500xL Genetic Analyzer (Applied Biosystems; Thermo Fisher Scientific, Inc.). STR profiling was performed using the GeneMapper Software (Applied Biosystems; Thermo Fisher Scientific, Inc.). The similarity of STRs among the reported cell lines was examined using the CLASTR function of Cellosaurus (18). Based on the Tanabe algorithm (19), the STR match ratio between the established cell lines and the corresponding original tumor was calculated. The score met the standard match threshold of $80 \%$ (19) based on Tanabe algorithms and non-empty marker mode that computed the score to handle cases in which allele data is missing for the query or the reference. Mycoplasma DNA was assessed in the tissue culture medium using an e-Myco Mycoplasma PCR Detection Kit (Intron Biotechnology).

Single nucleotide polymorphism (SNP) array. Copy number alterations were examined using an Infinium OmniExpressExome-8 v.1.4 BeadChip (Illumina, Inc.) following the manufacturer's protocol. Detailed descriptions of these procedures are provided in previous reports (20). Abnormal copy number regions were detected using the circular binary segmentation algorithm $(21,22)$ using the $\mathrm{R}$ package DNAcopy from Bioconductor (23). Amplifications were defined as regions for which the copy number was $>3$. Deletions were defined as regions of which $<1$ copy was present in the tumor cells. Among identified genes with copy number alterations, a search for 'cancer-related genes' was performed in the Cancer Gene Census in the Catalogue Of Somatic Mutations In Cancer database (GRCh 37 v.91) (24).

Histological evaluation. All specimens for $\mathrm{H} \& \mathrm{E}$ staining were cut into 4- $\mu \mathrm{m}$ slices and placed on the slides. After deparaffinization, the nuclei were immersed in hematoxylin staining solution (Muto Chemical) for $15 \mathrm{~min}$, followed by washing out the solution with tap water. Subsequently, cytoplasm and stromal matrix were stained with eosin (Muto Chemical). The slides 
were washed in water and mounted. Immunohistochemical staining was performed on formalin-fixed, paraffin-embedded specimens obtained through surgery and on NCC-DMM1-C1 cells. NCC-DMM1-C1 cells were detached by treatment with trypsin and cell suspensions were solidified using iPGell (Genostaff) according to the manufacturer's protocol. Cell masses were fixed with $10 \%$ formalin and embedded in paraffin. Cell blocks were cut into sections that were processed for immunolabeling and H\&E staining. Sections were incubated with antibodies against various proteins. The expression of cytokeratin, D2-40, HEG1 and WT1 was evaluated using the following primary antibodies: Cytokeratin AE1/3 (AE1/AE3; 1:200 dilution; cat. no. sc-81714; Santa Cruz Biotechnology, Inc.), D2-40 (D2-40; 1:200 dilution; cat. no. PDM558; Diagnostic Biosystem), sialylated heart development protein with EGF-like domains 1 (SKM9-2; prediluted; cat. no. 418231; Nichirei Biosciences, Inc.) and WT1 (6F-H2; M3561, 1:50 dilution; Agilent Technologies, Inc.). Antigen detection was performed using a Dako Autostainer and EnVision Detection System (Dako; Agilent Technologies, Inc.) according to the manufacturer's protocol. The slides were counterstained with hematoxylin.

Cell proliferation assay. The cells were seeded into 24-well culture plates at a density of $2.5 \times 10^{4}$ cells/well as described previously (17). The cell viability was monitored using Cell Counting Kit-8 (CCK-8; Dojindo Molecular Technologies, Inc.). The absorbance of each well at $450 \mathrm{~nm}$ was recorded at multiple time-points using a microplate reader (Bio-Rad Laboratories, Inc.). Growth curves were constructed by plotting the absorbance as a function of culture time and these were used to estimate the population doubling time. The cells were maintained in a humidified atmosphere of $5 \% \mathrm{CO}_{2}$ at $37^{\circ} \mathrm{C}$.

Spheroid formation assay. Spheroid formation was performed by placing $1 \times 10^{4}$ cells in a 96-well Clear Flat Bottom Ultra-Low Attachment Microplate (Corning, Inc.) in DMEM/F12 medium containing $10 \% \mathrm{FBS}$, as described previously (17). After three days of plating, spheroid formation was monitored using a BZ-9000 fluorescence microscope (Keyence). Spheroid formation assays were performed in duplicate.

Transwell cell invasion assay. The invasive capability of NCC-DMM1-C1 cells and MG63 osteosarcoma cells (The Japanese Collection of Research Bioresources) (25) was measured as previously described (17). Cells ( $1 \times 10^{5}$ and $\left.2 \times 10^{5}\right)$ were seeded in the upper chamber. Following incubation, the cells in the three separate areas were counted under a microscope (Keyence) at x100 magnification.

Tyrosine kinase activity assay. Tyrosine kinase activity was examined for multiple kinases using the PamChip TK peptide microarray system (PamGene International B.V.) as previously described (26). The protein lysate $(5 \mu \mathrm{g})$ was extracted from the tissue cultured cells and tumor tissue and hybridized to the membrane array, which included 144 peptides. The average signal intensity of the 144 hybridized peptides based on the end levels of the phosphorylation curve was used for the activity analysis. All data analyses were performed using BioNavigator v.6.3.67.0 (PamGene International B.V.).
Table I. Results of short tandem repeat analysis.

Allele number

STR locus

(chromosome)

NCC-DMM1-C1 Normal tissue

\begin{tabular}{lrr}
\hline Amelogenin (X Y) & X, Y & X, Y \\
TH01 (3) & 9 & 9 \\
D21S11 (21) & $29,32.2$ & $29,32.2$ \\
D5S818 (5) & 9,11 & 9,11 \\
D13S317 (13) & 12 & 12 \\
D7S820 (7) & 8 & 8 \\
D16S539 (16) & 9,12 & 9,12 \\
CSF1PO (5) & 12 & 12 \\
vWA (12) & 18,19 & 18,19 \\
TPOX (2) & 8,11 & 8,11 \\
\hline
\end{tabular}

Active kinases were predicted using PhosphoSitePlus (27), the UniProt database (28) and the Human Protein Reference Database (29).

Screening for anti-proliferative effects of anti-cancer agents. The anti-proliferative effects of 213 anti-cancer agents (Table SI) were examined using the CCK-8 assay, as described previously (17). The cells were seeded at $5 \times 10^{3}$ cells/well in DMEM/F12 medium supplemented with $10 \%$ FBS using the Bravo Automated Liquid Handling Platform (Agilent Technologies, Inc.). The following day, anti-cancer agent compounds (10 $\mu \mathrm{M}$; Selleck Chemicals) and 0.1\% DMSO were added using the Bravo Automated Liquid Handler. After $72 \mathrm{~h}$, survival rates were assessed using the CCK- 8 reagent according to the manufacturer's protocol. The response rate was calculated relative to that of the DMSO control. Dose-response experiments were performed to validate the candidate anti-cancer agents that were identified during the pilot screening. The compounds were dispensed into 384-well plates with serial dilution at 10 different concentrations, ranging from 0.1 to $100,000 \mathrm{nM}$, using an Echo 555 Acoustic Liquid Handler (Labcyte Inc.) and $\mathrm{IC}_{50}$ values were determined. CCK-8 absorbance was measured using an Epoch multimode multiplate reader (BioTek Instruments). Absorbance values were plotted as a function of the compound concentrations to obtain $\mathrm{IC}_{50}$ values using GraphPad Prism 8.1.0 software (GraphPad Inc.). This screening was performed in duplicate.

\section{Results}

Authentication and quality control of the established cell line. NCC-DMM1-C1 was maintained for $>16$ months and passaged $>30$ times under tissue culture conditions. The characterization of NCC-DMM1-C1 cells was performed by STR profiling at the 20th passage. The same patterns of the peaks in all loci composed of D5S818, D13S317, D7S820, D16S539, vWA, TH01, TP0X, CSF1P0, and the sex chromosomal marker amelogenin were observed in both the normal tissues and the NCC-DMM1-C1 cells. The normal tissues were composed of the adjacent tissue to the tumor tissue which did not include the 

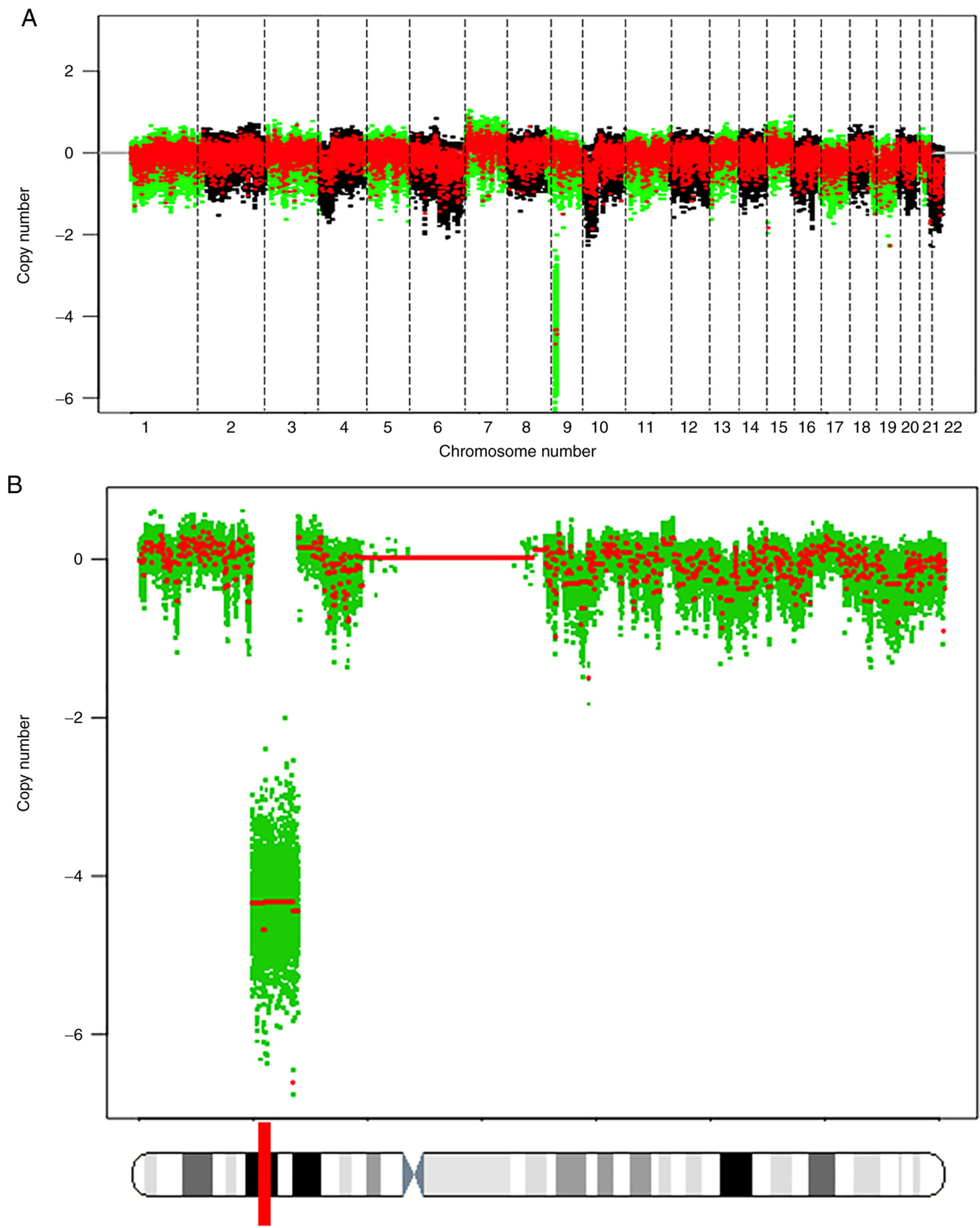

Figure 2. Single nucleotide polymorphism genotyping of NCC-DMM1-C1 cells. (A and B) The chromosome numbers are displayed on the x-axis; chromosomes 1 to 22 are presented from left to right using green and black colors, and chromosomal segments are displayed in red dots. The copy number values are indicated on the y-axis. Multiple deletions were identified in NCC-DMM1-C1 cells. (B) Cytogenetic band of chromosome 9 and the copy number variation data of chromosome 9. The genomic positions are indicated on the $\mathrm{x}$-axis and the copy number values are indicated on the $y$-axis. The red dots indicate chromosomal segments. The red bar indicates the corresponding site of cyclin-dependent kinase inhibitor $2 \mathrm{~A}$.

tumor cells near the chest wall. The authentication indicated that the normal tissues and the NCC-DMM1-C1 cells were derived from the same origin (Table I, Fig. S1). Calculated based on the Tanabe algorithm (19), the STR match ratio between the NCC-DMM1-C1 cells and the corresponding original tumor was $100 \%$. The score met the standard match 

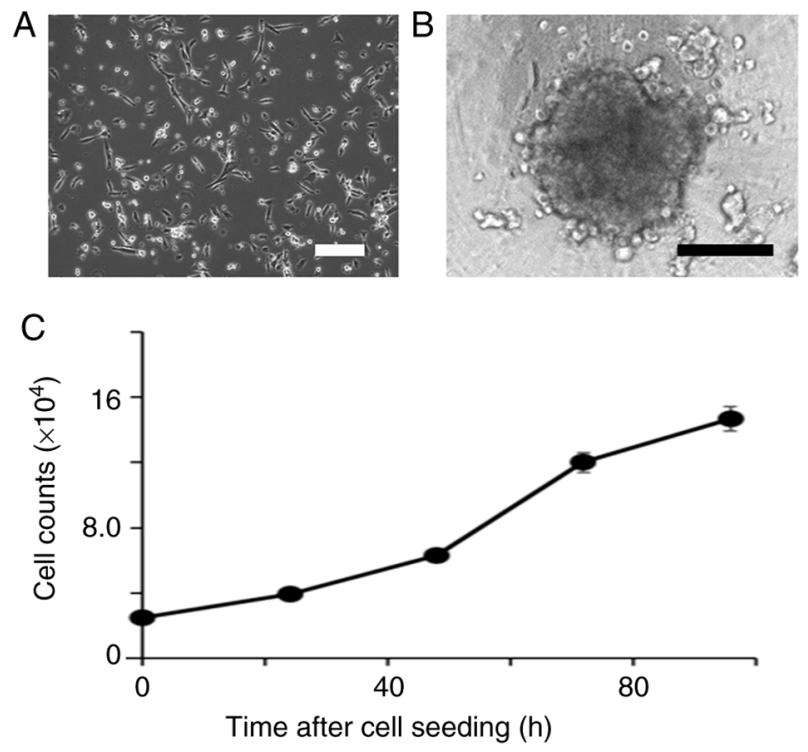

D

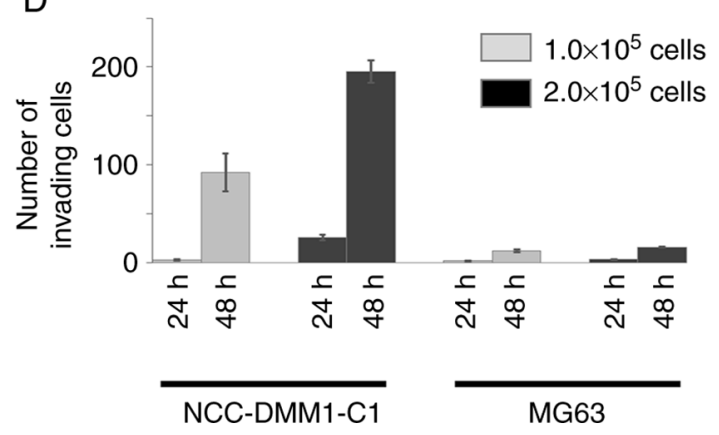

Figure 3. Characterization of NCC-DMM1-C1 cells. (A) Spindle-shaped morphology of NCC-DMM1-C1 cells. (B) Spheroid formation of NCC-DMM1-C1 cells in 96-well spheroid microplates (scale bars, $200 \mu \mathrm{m}$ ). (C) Growth curve of NCC-DMM1-C1 cells. Each datapoint represents a mean of the number of cells $(n=3)$. (D) Invasion ability of NCC-DMM1-C1 cells and osteosarcoma MG63 cells. Data are presented as mean \pm standard deviation.

threshold of $80 \%$ (19). According to Cellosaurus, the profile did not match that of cell lines deposited in public cell banks. It was therefore indicated that NCC-DMM1-C1 is a novel cell line. The DNA sequence of Mycoplasma was not detected in the tissue culture medium (data not shown) and it was concluded that NCC-DMM1-C1 was not contaminated with Mycoplasma.

Cell line characteristics. SNP array experiments revealed the presence of copy number variants mostly involving partial deletions of chromosomal arms (9p) (Fig. 2A) and cyclin-dependent kinase inhibitor 2A was located in the loci of deletions (Fig. 2B). NCC-DMM1-C1 cells exhibited a polygonal or spindle-shaped cytoplasm, arranged in a partially cohesive sheet-like structure, with enlarged and variably sized nuclei (Fig. 3A). The NCC-DMM1-C1 cells formed spheroids in low-attachment substrates (Fig. 3B) and exhibited constant growth (Fig. 3C). The population doubling time based on the growth curve was $25.9 \mathrm{~h}$ (Fig. 3C). The invasion capability of NCC-DMM1-C1 cells was higher than that of MG63 osteosarcoma cells (Figs. 3D and S2).

Histological evaluation. H\&E staining of the sectioned tissue indicated that the spindle-shaped cells had a storiform pattern
A
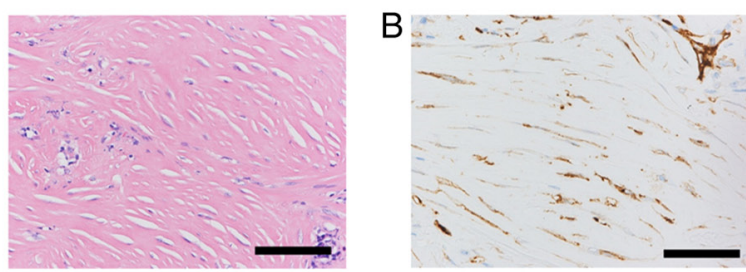

C
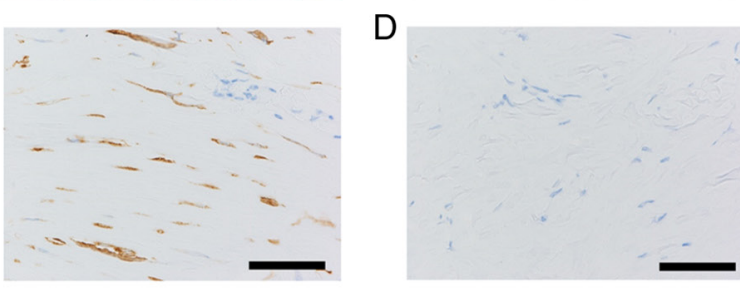

E

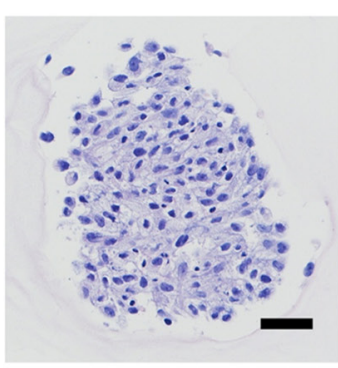

F

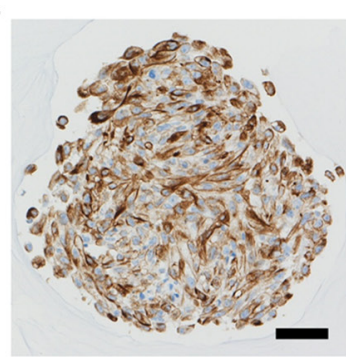

G

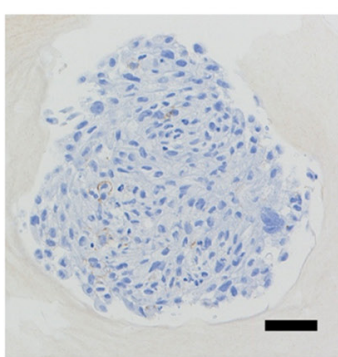

$\mathrm{H}$
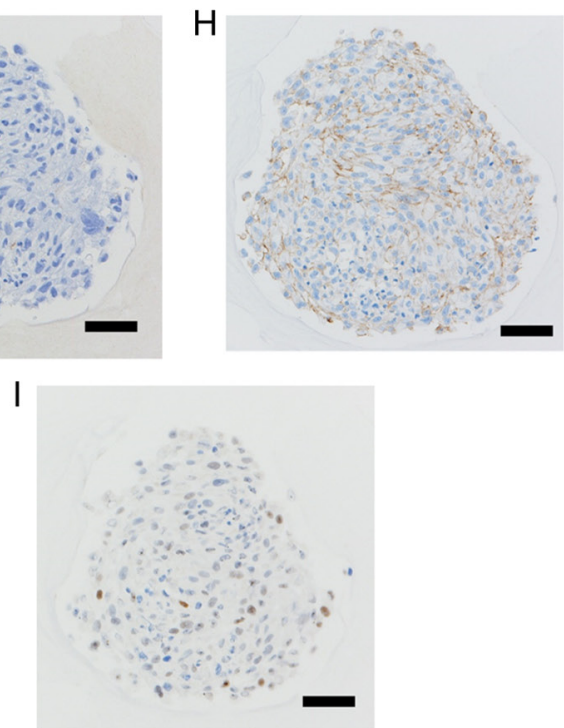

Figure 4. Histological evaluation. (A) Representative hematoxylin \& eosin-stained section of the original tumor from which the NCC-DMM1-C1 cell line was established. Immunohistochemistry demonstrated positive expression of (B) cytokeratin AE1/3 and (C) D2-40, and negative expression of (D) WT1. (E) Representative hematoxylin \& eosin-stained section of NCC-DMM1-C1 cells. (F) Diffuse positive immunostaining for cytokeratin AE1/3. (G) Focally positive immunostaining for D2-40. (H) Weak but diffuse positive immunostaining for HEG1. (I) Mostly positive immunostaining for WT1 (scale bars, $200 \mu \mathrm{m}$ ). HEG1, heart development protein with EGF-like domains 1.

and proliferated densely (Fig. 4A). Immunohistochemistry revealed diffusely positive immunoreactivity for cytokeratin AE1/AE3 (Fig. 4B) and D2-40 (Fig. 4C) and negative immunoreactivity for WT1 (Fig. 4D). H\&E staining of the NCC-DMM1-C1 cells indicated the same pattern as that of the tissue section with a storiform pattern and proliferated density and the composition of one type of cells, i.e. tumor cells with no other cells (Fig. 4E). Immunohistochemistry revealed diffusely 
Table II. Summary of $\mathrm{IC}_{50}$ values.

\begin{tabular}{llc}
\hline CAS no. & \multicolumn{1}{c}{ Name of drug } & $\mathrm{IC}_{50}(\mu \mathrm{M})$ \\
\hline $15663-27-1$ & Cisplatin & 5.38 \\
$95058-81-4$ & Gemcitabine & 0.053 \\
$901119-35-5$ & Fostamatinib & 0.097 \\
$443913-73-3$ & Vandetanib & 13.75 \\
$366017-09-6$ & Mubritinib & 0.43 \\
$179324-69-7$ & Bortezomib & 0.055 \\
$26833-87-4$ & Homoharringtonine & 0.072 \\
$65271-80-9$ & Mitoxantrone & 0.26 \\
$267243-28-7$ & Canertinib & 0.76 \\
$357166-30-4$ & Pemetrexed & 0.49 \\
$149647-78-9$ & disodium hydrate & 1.26 \\
$71486-22-1$ & Vorinostat & 0.04 \\
$252916-29-3$ & Vinorelbine & 30.06
\end{tabular}

$\mathrm{IC}_{50}$, half-maximal inhibitory concentration.

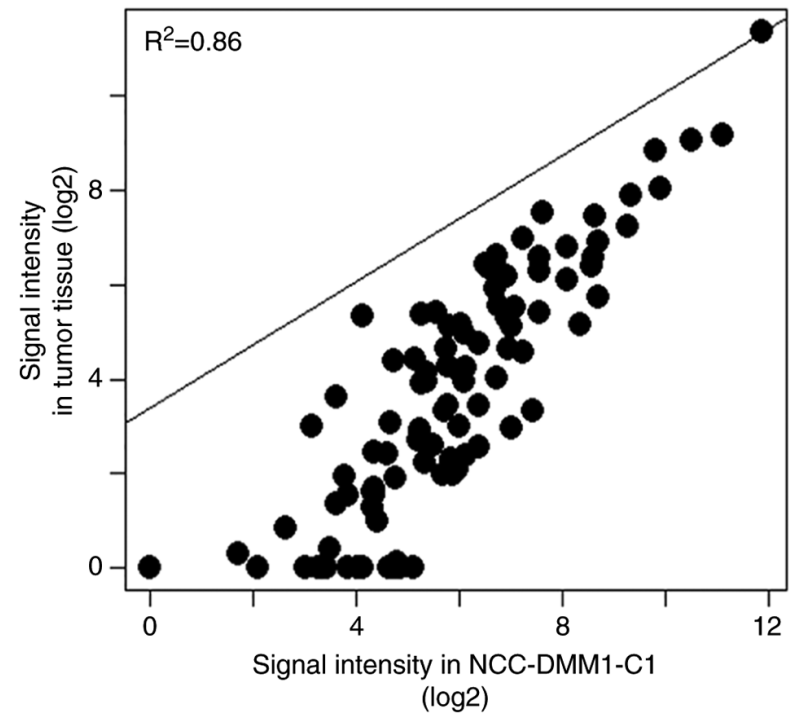

Figure 5. Kinase activity profiling of desmoplastic malignant pleural mesothelioma. Correlation analysis of tyrosine kinase activity between NCC-DMM1-C1 cells and tumor tissue.

positive staining for cytokeratin AE1/AE3 (Fig. 4F), negativity for D2-40 (Fig. 4G), weakly but diffusely positive staining for HEG1 (Fig. 4H) and mostly positive staining for WT1 (Fig. 4I).

Kinase activity assay. Pearson's correlation scatter plot revealed high similarity of kinase activity between NCC-DMM1-C1 cells and tumor tissue $\left(\mathrm{r}^{2}=0.86\right.$; Fig. 4 , Table SII). The 25 most highly phosphorylated peptides were identified in both NCC-DMM1-C1 cells and tumor tissue (Table SIII). These peptides included FES, Wee1, platelet-derived growth factor receptor (PDGFR)- $\beta$ and Src (Table SIII).

Sensitivity to anti-cancer agents. The $\mathrm{IC}_{50}$ values of 13 of the anti-cancer agents applied were calculated, including four anti-cancer agents used for DMM and nine drugs that had relatively high inhibitory effects on cancer cell proliferation at $10 \mu \mathrm{M}$ (Fig. S3, Table SIV). The calculated $\mathrm{IC}_{50}$ values are summarized in Table II and the representative growth curves of five anti-cancer agents with $\mathrm{IC}_{50}$ values $<100 \mathrm{nM}$ (bortezomib, gemcitabine, homoharringtonine, fostamatinib and vinorelbine) are displayed in Fig. 5.

\section{Discussion}

DMM is an aggressive neoplasm with poor prognosis. The effects of surgery, chemotherapy and radiotherapy have remained to be fully defined. Thus, it is necessary to improve the current knowledge regarding the molecular mechanisms underlying disease progression of DMM and develop assay systems to evaluate novel anti-cancer agents. However, due to the rarity of the disease, only a small number of DMM cell lines have been reported and cell lines and xenografts for DMM are not readily available from public cell banks. The present study reported on the establishment a novel DMM cell line, designated as NCC-DMM1-C1 and determined its characteristics. Furthermore, anti-cancer drugs were screened using NCC-DMM1-C1 cells to evaluate whether the cell line may be used to screen drug candidates or not.

NCC-DMM1-C1 cells exhibited a spindle cell morphology and demonstrated constant growth and aggressive invasion, which reflect the characteristics of the original tumor. The spheroid formation capability contributes to the understanding of the behavior of DDM in a 3D environment. While NCC-DMM1-C1 cells had these features reflecting the original tumor, the immunohistochemical staining for D2-40 and WT1 was different between the tumor tissue and NCC-DMM1-C1 cells. NCC-DMM1-C1 cells stained negative for D2-40 expression and the tumor tissue demonstrated positive staining for D2-40 expression. Furthermore, NCC-DMM1-C1 cells demonstrated mostly positive WT1 expression and the tumor tissue demonstrated negative WT1 expression. Tumor tissue of DMM is composed of collagen tissue occupying $>50 \%$ of the tumor and tumor cells (2) and NCC-DMM1-C1 was composed of tumor cells only. In addition, the expression of D2-40 and WT1 in NCC-DMM1-C1 cells was different from that of the tumor tissue due to heterogeneity. The cell components and the heterogeneity may have led to the distinct differences in D2-40 and WT1 expression.

The drug screening assay identified bortezomib, gemcitabine, homoharringtonine, fostamatinib and vinorelbine as anti-cancer agents with low $\mathrm{IC}_{50}$ values in NCC-DMM1-C1. Gemcitabine is a DNA-damaging agent and has been investigated as a first-line single treatment in chemotherapy-naive patients or as a second- or third-line combination therapy in patients with MPM $(8,30,31)$. Vinorelbine is a microtubule-damaging agent and its efficacy has been reported in patients with progressive disease after treatment with pemetrexed-platinum chemotherapy $(8,31,32)$. Bortezomib is a proteasome inhibitor and its cytotoxic effects were revealed in a preclinical study using six cell lines of MPM (33). However, two subsequent Phase II studies did not reach the desired endpoint in MPM $(34,35)$. Further clinical trials are necessary to clarify the clinical utility of these three anti-cancer drugs for DMM. Homoharringtonine, a natural plant alkaloid extracted from Cephalotaxus harringtonia used 

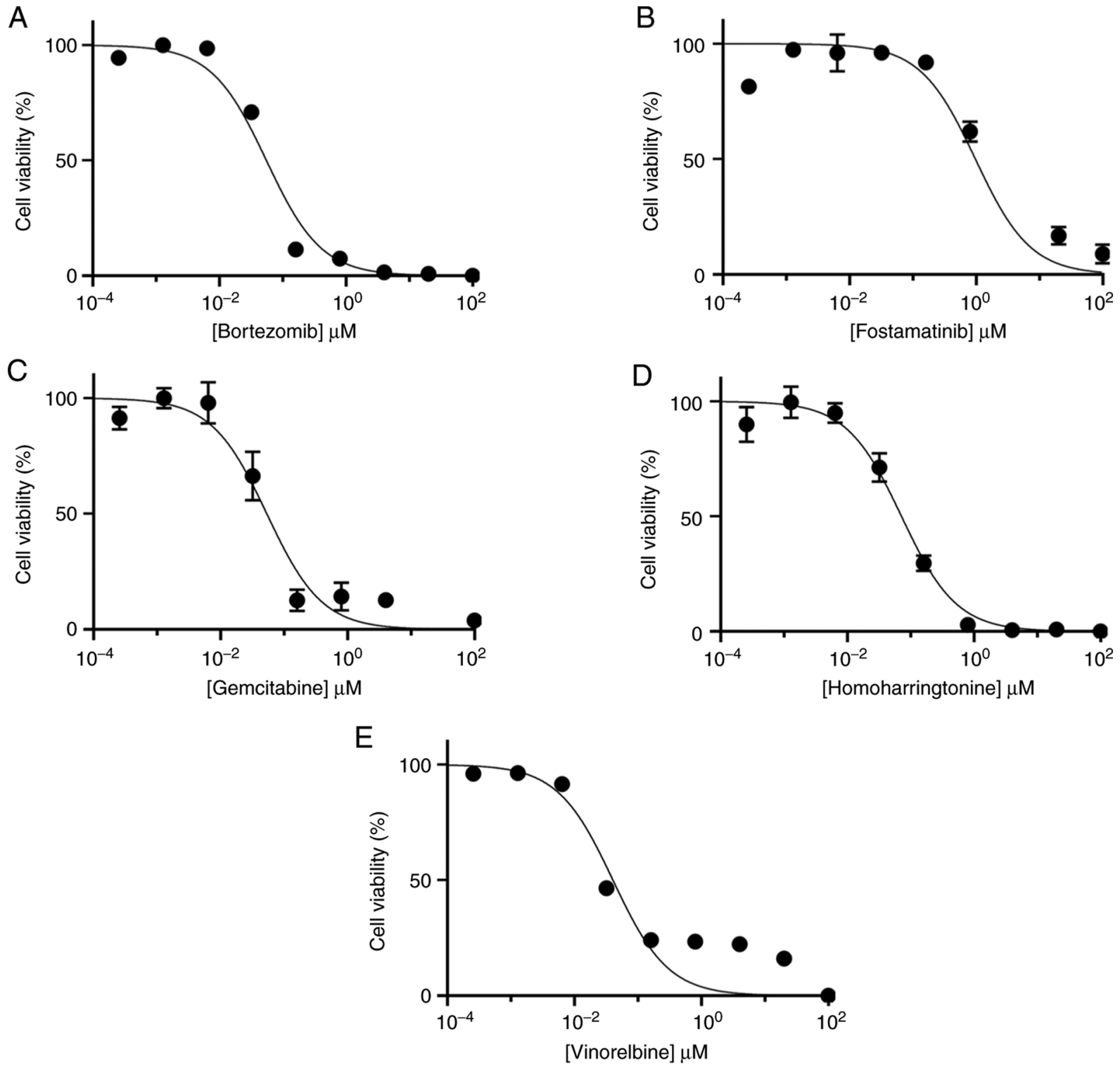

Figure 6. Growth curves of NCC-DMM1-C1 cells treated with anti-cancer agents. The proliferation of NCC-DMM1-C1 cells treated with anti-cancer agents, which had marked anti-proliferative effects at a fixed concentration, was assessed using growth curves. (A) Bortezomib, (B) fostamatinib, (C) gemcitabine, (D) homoharringtonine and (E) vinorelbine. Data are presented as mean \pm standard deviations based on two biological replicates.

in Chinese Traditional Medicine (36), has efficient inhibitory activity against myelocytic leukemia $(37,38)$. Fostamatinib is a pro-drug inhibitor of spleen tyrosine kinase, which is a key mediator of Fc and B-cell receptor signaling in inflammatory cells and has efficacy in non-Hodgkin lymphoma and chronic lymphocytic leukemia (39). The treatment utility of homoharringtonine and fostamatinib in MPM has not been previously reported and it is worth elucidating their effects on MPM using NCC-DMM1-C1 cells.

Comprehensive kinase activity assays revealed that kinase activity was similar between NCC-DMM1-C1 cells and the original tumor tissue, suggesting that the NCC-DMM1-C1 cell line may be useful for examining the effects of kinase inhibitors in vitro. It was also determined that the kinases FES, Wee1, PDGFR- $\beta$ and Src were highly activated in both NCC-DMM1-C1 cells and the tumor tissue. Tsao et al (40) demonstrated that Src was expressed and activated in MPM cell lines and 46 MPM tumor specimens. Additionally, the
MPM cell lines were sensitive to an Src inhibitor, as determined using in vitro cytotoxicity assays. Weel is a tyrosine kinase that phosphorylates and inactivates cyclin-dependent kinase 1 and is involved in G2 checkpoint signaling (41). Xu et al (41) reported that a kinome-wide CRISPR/Cas9 knockout screen identified Wee1, the loss of function of which sensitizes cells to standard combination cisplatin and pemetrexed chemotherapy in MPM cell lines and PDXs. Several groups have reported that the PDGF/PDGFR pathway is involved in mesothelioma carcinogenesis $(42,43)$. Melaiu et al $(44)$ reported that, in MPM cells, PDGFRB silencing causes a decrease in the proliferation rate and reduces the colony formation capacity, which highlights the utility of PDGFR- $\beta$ as a drug target. In the present study, high FES activity was observed, which has not been previously reported in MPM, to the best of our knowledge. Experiments to evaluate kinase activity cannot be perfect and to compensate for the drawbacks of each experiment, it is required to use different types of experiments prior 
to clinical trials. Therefore, kinase activities identified in the present study need to be validated using other methods such as western blot analysis. The present findings confirm that kinase activity may be used as a drug target and biomarker to assess the utility of kinase inhibitors.

In conclusion, in the present study, a novel cell line was established from tumor tissue of DMM, designated as NCC-DMM1-C1, and its characteristics and utility in high-throughput drug screening were demonstrated. However, the results of the present study were obtained from only a single cell line; further cell lines from different patients are required to generate reliable results. In addition, the present results regarding the candidate anti-cancer drugs should be validated in other patient-derived cancer models, including organoids and xenografts, prior to clinical trials. In addition, the candidate anti-cancer drugs were identified using the drug screening based on a single agent. Reflecting the practical clinical situation, drug screening based on several agents of the combination treatment will be performed in the future. Furthermore, evaluation of highly phosphorylated kinases and the relevant molecular pathway should be investigated by other proteomics techniques such as mass spectrometry. The present findings, along with future validations, will provide a deeper understanding of DMM, suggesting the possible utility of NCC-DMM1-C1 cells in drug development.

\section{Acknowledgements}

The authors appreciate the technical support provided by Miss Yu Kuwata (Division of Rare Cancer Research, National Cancer Center, Tokyo, Japan).

\section{Funding}

This research was supported by the Japan Agency for Medical Research and Development (grant no. 20ck0106537h0001).

\section{Availability of data and materials}

The cell line of the current study is available from the corresponding author on reasonable request. The datasets generated during the current study are available from the Gene Expression Omnibus (GEO) repository (45). The present data of the peptide microarray are accessible through GEO (series accession no. GSE185107; https://www.ncbi.nlm.nih. gov/geo/query/acc.cgi?acc=GSE185107) and the data of the SNP array are accessible through GEO (series accession no. GSE185549; https://www.ncbi.nlm.nih.gov/geo/query/acc. cgi?acc=GSE185549)

\section{Authors' contributions}

RN and YYoshimatsu established the cell line. NM and YYa performed the pathological diagnoses and immunohistochemical staining. SW, YYoshida and AS prepared tumor tissues and obtained clinical information of the donor patient. RN, YYoshimatsu and TO performed all experiments, including authentication, drug screening assay and kinase assay. TK was responsible for study conception and design. RN, Y Yoshimatsu and TK prepared the manuscript, including the figures and tables. RN, YYoshimatsu and TK edited the manuscript. All authors read and approved the final manuscript prior to submission. RN and TK checked and confirm the authenticity of all the raw data.

\section{Ethics approval and consent to participate}

The use of clinical materials for this study was approved by the ethics committee of the National Cancer Center (approval no. 2015-108) and written informed consent was obtained from the patient. The informed consent was for collection of tissues from the patient for the present study and publication of data obtained using the tissues. All procedures followed were in accordance with the ethical standards of the responsible committee on human experimentation (institutional and national) and with the Helsinki Declaration.

\section{Patient consent for publication}

Although detailed clinical data were not included in the present study, consent for publication was obtained from the patient included in the present study.

\section{Competing interests}

The authors declare that they have no competing interests.

\section{References}

1. Gibbs AR and Thunnissen FB: Histological typing of lung and pleural tumours: Third edition. J Clin Pathol 54: 498-499, 2001.

2. Galateau-Salle F, Churg A, Roggli V and Travis WD; World Health Organization Committee for Tumors of the Pleura: The 2015 World Health Organization classification of tumors of the pleura: Advances since the 2004 classification. J Thorac Oncol 11: 142-154, 2016.

3. Hashimoto K, Okuma Y, Hosomi Y and Hishima T: Malignant mesothelioma of the pleura with desmoplastic histology: A case series and literature review. BMC Cancer 16: 718, 2016.

4. Leung AN, Müller NL and Miller RR: CT in differential diagnosis of diffuse pleural disease. AJR Am J Roentgenol 154: 487-492, 1990

5. Kawashima A and Libshitz HI: Malignant pleural mesothelioma: CT manifestations in 50 cases. AJR Am J Roentgenol 155: 965-969, 1990.

6. Wang ZJ, Reddy GP, Gotway MB, Higgins CB, Jablons DM, Ramaswamy M, Hawkins RA and Webb WR: Malignant pleural mesothelioma: Evaluation with CT, MR imaging, and PET. Radiographics 24: 105-119, 2004.

7. Vogelzang NJ, Rusthoven JJ, Symanowski J, Denham C, KaukelE, Ruffie P, Gatzemeier U, Boyer M, Emri S, Manegold C, et al: Phase III study of pemetrexed in combination with cisplatin versus cisplatin alone in patients with malignant pleural mesothelioma. J Clin Oncol 21: 2636-2644, 2003.

8. Zauderer MG, Kass SL, Woo K, Sima CS, Ginsberg MS and Krug LM: Vinorelbine and gemcitabine as second- or third-line therapy for malignant pleural mesothelioma. Lung Cancer 84: 271-274, 2014.

9. Christoph DC and Eberhardt WE: Systemic treatment of malignant pleural mesothelioma: New agents in clinical trials raise hope of relevant improvements. Curr Opin Oncol 26: 171-181, 2014.

10. Sharma SV, Haber DA and Settleman J: Cell line-based platforms to evaluate the therapeutic efficacy of candidate anticancer agents. Nat Rev Cancer 10: 241-253, 2010.

11. Barretina J, Caponigro G, Stransky N, Venkatesan K, Margolin AA, Kim S, Wilson CJ, Lehár J, Kryukov GV, Sonkin D, et al: The cancer cell line encyclopedia enables predictive modelling of anticancer drug sensitivity. Nature 483: 603-607, 2012. 
12. Iorio F, Knijnenburg TA, Vis DJ, Bignell GR, Menden MP, Schubert M, Aben N, Gonçalves E, Barthorpe S, Lightfoot $\mathrm{H}$, et al: A landscape of pharmacogenomic interactions in cancer. Cell 166: 740-754, 2016.

13. Teicher BA, Polley E, Kunkel M, Evans D, Silvers T, Delosh R, Laudeman J, Ogle C, Reinhart R, Selby M, et al: Sarcoma cell line screen of oncology drugs and investigational agents identifies patterns associated with gene and microRNA expression. Mol Cancer Ther 14: 2452-2462, 2015.

14. Oey H, Daniels M, Relan V, Chee TM, Davidson MR, Yang IA, Ellis JJ, Fong KM, Krause L and Bowman RV: Whole-genome sequencing of human malignant mesothelioma tumours and cell lines. Carcinogenesis 40: 724-734, 2019.

15. Relan V, Morrison L, Parsonson K, Clarke BE, Duhig EE, Windsor MN, Matar KS, Naidoo R, Passmore L, McCaul E, et al: Phenotypes and karyotypes of human malignant mesothelioma cell lines. PLoS One 8: e58132, 2013.

16. Conte N, Mason JC, Halmagyi C, Neuhauser S, Mosaku A, Yordanova G, Chatzipli A, Begley DA, Krupke DM Parkinson H, et al: PDX Finder: A portal for patient-derived tumor xenograft model discovery. Nucleic Acids Res 47 (D1): D1073-D1079, 2019.

17. Yoshimatsu Y, Noguchi R, Tsuchiya R, Kito F, Sei A, Sugaya J, Nakagawa M, Yoshida A, Iwata S, Kawai A and Kondo T: Establishment and characterization of NCC-CDS2-C1: A nove patient-derived cell line of CIC-DUX4 sarcoma. Hum Cell 33 427-436, 2020.

18. Bairoch A: The cellosaurus, a cell-line knowledge resource. J Biomol Tech 29: 25-38, 2018

19. Capes-Davis A, Reid YA, Kline MC, Storts DR, Strauss E, Dirks WG, Drexler HG, MacLeod RA, Sykes G, Kohara A, et al: Match criteria for human cell line authentication: Where do we draw the line? Int J Cancer 132: 2510-2519, 2013.

20. Noguchi R, Yoshimatsu Y, Ono T, Sei A, Hirabayashi K, Ozawa I, Kikuta $\mathrm{K}$ and Kondo T: Establishment and characterization of NCC-PLPS1-C1, a novel patient-derived cell line of pleomorphic liposarcoma. Hum Cell 34: 688-697, 2020.

21. Olshen AB, Venkatraman ES, Lucito R and Wigler M: Circular binary segmentation for the analysis of array-based DNA copy number data. Biostatistics 5: 557-572, 2004.

22. Venkatraman ES and Olshen AB: A faster circular binary segmentation algorithm for the analysis of array CGH data. Bioinformatics 23: 657-663, 2007.

23. Willenbrock $\mathrm{H}$ and Fridlyand J: A comparison study: Applying segmentation to array CGH data for downstream analyses. Bioinformatics 21: 4084-4091, 2005.

24. Forbes SA, Tang G, Bindal N, Bamford S, Dawson E, Cole C, Kok CY, Jia M, Ewing R, Menzies A, et al: COSMIC (the catalogue of somatic mutations in cancer): A resource to investigate acquired mutations in human cancer. Nucleic Acids Res 38 (Database Issue): D652-D657, 2010.

25. Billiau A, Edy VG, Heremans H, Van Damme J, Desmyter J, Georgiades JA and De Somer P: Human interferon: Mass production in a newly established cell line, MG-63. Antimicrob Agents Chemother 12: 11-15, 1977.

26. Sikkema AH, Diks SH, den Dunnen WF, ter Elst A, Scherpen FJ, Hoving EW,Ruijtenbeek R, Boender PJ, de Wijn R,Kamps WA, et al: Kinome profiling in pediatric brain tumors as a new approach for target discovery. Cancer Res 69: 5987-5995, 2009.

27. Hornbeck PV, Zhang B, Murray B, Kornhauser JM, Latham V and Skrzypek E: PhosphoSitePlus, 2014: Mutations, PTMs and recalibrations. Nucleic Acids Res 43 (Database Issue): D512-D520, 2015.

28. UniProt Consortium: UniProt: A worldwide hub of protein knowledge. Nucleic Acids Res 47 (D1): D506-D515, 2019.

29. Keshava Prasad TS, Goel R, Kandasamy K, Keerthikumar S, Kumar S, Mathivanan S, Telikicherla D, Raju R, Shafreen B, Venugopal A, et al: Human protein reference database-2009 update. Nucleic Acids Res 37 (Database Issue): D767-D772, 2009.

30. van Meerbeeck JP, Baas P, Debruyne C, Groen HJ, Manegold C, Ardizzoni A, Gridelli C, van Marck EA, Lentz M and Giaccone G: A Phase II study of gemcitabine in patients with malignant pleural mesothelioma. European organization for research and treatment of cancer lung cancer cooperative group. Cancer 85: 2577-2582, 1999.
31. Zucali PA, Ceresoli GL, Garassino I, De Vincenzo F, Cavina R, Campagnoli E, Cappuzzo F, Salamina S, Soto Parra HJ and Santoro A: Gemcitabine and vinorelbine in pemetrexed-pretreated patients with malignant pleural mesothelioma. Cancer 112: 1555-1561, 2008.

32. Zucali PA, Perrino M, Lorenzi E, Ceresoli GL, De Vincenzo F, Simonelli M, Gianoncelli L, De Sanctis R, Giordano L and Santoro A: Vinorelbine in pemetrexed-pretreated patients with malignant pleural mesothelioma. Lung Cancer 84: 265-270, 2014.

33. Szulkin A, Nilsonne G, Mundt F, Wasik AM, Souri P, Hjerpe A and Dobra K: Variation in drug sensitivity of malignant mesothelioma cell lines with substantial effects of selenite and bortezomib, highlights need for individualized therapy. PLoS One 8: e65903, 2013.

34. O'Brien ME, Gaafar RM, Popat S, Grossi F, Price A, Talbot DC, Cufer T, Ottensmeier C, Danson S, Pallis A, et al: Phase II study of first-line bortezomib and cisplatin in malignant pleural mesothelioma and prospective validation of progression free survival rate as a primary end-point for mesothelioma clinical trials (European organisation for research and treatment of cancer 08052). Eur J Cancer 49: 2815-2822, 2013

35. Fennell DA, McDowell C, Busacca S, Webb G, Moulton B, Cakana A, O'Byrne KJ, Meerbeeck JV, Donnellan P, McCaffrey J and Baas P: Phase II clinical trial of first or second-line treatment with bortezomib in patients with malignant pleural mesothelioma. J Thorac Oncol 7: 1466-1470, 2012.

36. Huang CC, Han CS, Yue XF, Shen CM, Wang SW, Wu FG and $\mathrm{Xu}$ B: Cytotoxicity and sister chromatid exchanges induced in vitro by six anticancer drugs developed in the People's Republic of China. J Natl Cancer Inst 71: 841-847, 1983.

37. Kantarjian HM, Keating MJ, Walters RS, Koller CA, McCredie KB and Freireich EJ: Phase II study of low-dose continuous infusion homoharringtonine in refractory acute myelogenous leukemia. Cancer 63: 813-817, 1989.

38. Quintás-Cardama A, Kantarjian H, Garcia-Manero G, O'Brien S, Faderl S, Estrov Z, Giles F, Murgo A, Ladie N, Verstovsek S and Cortes J: Phase I/II study of subcutaneous homoharringtonine in patients with chronic myeloid leukemia who have failed prior therapy. Cancer 109: 248-255, 2007.

39. Friedberg JW, Sharman J, Sweetenham J, Johnston PB, Vose JM, Lacasce A, Schaefer-Cutillo J, De Vos S, Sinha R, Leonard JP, et al: Inhibition of Syk with fostamatinib disodium has significant clinical activity in non-Hodgkin lymphoma and chronic lymphocytic leukemia. Blood 115: 2578-2585, 2010.

40. Tsao AS, He D, Saigal B, Liu S, Lee JJ, Bakkannagari S, Ordonez NG, Hong WK, Wistuba I and Johnson FM: Inhibition of c-Src expression and activation in malignant pleural mesothelioma tissues leads to apoptosis, cell cycle arrest, and decreased migration and invasion. Mol Cancer Ther 6: 1962-1972, 2007.

41. Xu D, Liang SQ, Yang H, Bruggmann R, Berezowska S, Yang Z, Marti TM, Hall SRR, Gao Y, Kocher GJ, et al: CRISPR screening identifies WEE1 as a combination target for standard chemotherapy in malignant pleural mesothelioma. Mol Cancer Ther 19: 661-672, 2020 .

42. Dorai T, Kobayashi H, Holland JF and Ohnuma T: Modulation of platelet-derived growth factor-beta mRNA expression and cell growth in a human mesothelioma cell line by a hammerhead ribozyme. Mol Pharmacol 46: 437-444, 1994.

43. Langerak AW, Dirks RP and Versnel MA: Splicing of the platelet-derived-growth-factor A-chain mRNA in human malignant mesothelioma cell lines and regulation of its expression. Eur J Biochem 208: 589-596, 1992.

44. Melaiu O, Catalano C, De Santi C, Cipollini M, Figlioli G, Pellè L, Barone E, Evangelista M, Guazzelli A, Boldrini L, et al: Inhibition of the platelet-derived growth factor receptor beta (PDGFRB) using gene silencing, crenolanib besylate, or imatinib mesylate hampers the malignant phenotype of mesothelioma cell lines. Genes Cancer 8: 438-452, 2017.

45. Edgar R, Domrachev M and Lash AE: Gene expression omnibus: NCBI gene expression and hybridization array data repository. Nucleic Acids Res 30: 207-210, 2002.

This work is licensed under a Creative Commons Attribution-NonCommercial-NoDerivatives 4.0 International (CC BY-NC-ND 4.0) License. 